\title{
Adaptive high-Q bandpass photonic RF filter
}

\author{
Rong Zheng, Kamal E. Alameh, Zhenglin Wang \\ Centre for MicroPhotonic Systems, Electron Science Research Institute \\ Edith Cowan University, 100 Joondalup Drive, Joondalup, Australia 6027
}

\begin{abstract}
In this paper, a MicroPhotonic-based high-Q tunable RF filter architecture is proposed. The architecture uses a Vertical Cavity Surface Emitting Laser (VCSEL) array, a 2D ultra-wideband photo-receiver array and a multi-cavity optical substrate to generate a large number of optical true-time delays thus achieving arbitrary, high-resolution RF filter transfer characteristics. By tuning the responses of the different optical cavities, adaptive high-Q RF filter characteristics can be realized over a wide RF frequency range. Proof-of-concept experimental results demonstrate the adaptability of the MicroPhotonic-based RF filter.
\end{abstract}

Keywords: Photonic RF filter, adaptive filtering, MicroPhotonics.

\section{INTRODUCTION}

Photonic adaptive RF signal processing has attracted a lot of attention recently [1, 2]. It offers many advantages over conventional electronic RF signal processors, including almost no limitation in frequency bandwidth, low loss and immunity to electromagnetic interference. The realization of a high-Q tunable RF filter is very important in applications such as RF radars, telescopes and RF wireless systems [3, 4]. Many promising approaches to achieve high-Q bandpass photonic RF filter have been proposed [5,6]. Among various implementations, optical amplifiers with a pair of fibre Bragg-gratings to achieve high-Q factor have been reported [5]. However, these structures are (i) limited to lowfrequency operations because of substantial phased-induced intensity noise introduced by the high-coherence laser sources in optical communication system, and (ii) their transfer characteristics cannot be arbitrarily reconfigured. To overcome these limitations, Capmany et al. proposed the use of multiple wavelength sources for generating reconfigurable RF characteristics [7], but their technique becomes impractical for high-resolution filter characteristics, because it requires a large number of wavelengths of accurate separation and dynamically-apodized Bragg gratings to control the power levels of each wavelength source component. Recently, an 8x8 true-time delay module that integrates holographic-grating couplers and graded index (GRIN) lenses on top of optical substrates, has been proposed for K-band phased array antenna beamforming [8]. However, coupling the delayed optical signals into optical fibers limits the maximum number of delays that can be synthesized, hence limiting the maximum Q-factor of the RF filter.

In this paper, a MicroPhotonic RF high-Q filter structure is proposed, in which a Vertical Cavity Surface Emitting Laser (VCSEL) array, a 2D ultra-wideband photoreceiver array and a multi-cavity optical substrate are integrated to achieve adaptive high-Q RF filter transfer characteristics with high-frequency operation range. Each optical cavity generates a number of delayed RF-modulated optical signals. By having many optical cavities of different lengths, a large number of different delayed RF-modulated optical signals can be produced, whose amplitudes can be independently adjusted by varying the gain profile of the photo-receiver array. By combining the delayed RF signals, an adaptive Finite Impulse Response (FIR) transversal filter can be realized. Simulations results show that a MicroPhotonic structure employing a $32 \times 32$ photoreceiver array can realize a high-Q adaptive RF filter with $\mathrm{Q}$ factor as high as 500 and its working frequency can be tuned up to $5 \mathrm{GHz}$. A proof-of-concept 4-tap MicroPhotonic RF filter structure is experimentally demonstrated, and results show excellent agreement between simulation and meausrement. 


\section{MICROPHOTONIC HIGH-Q RF FILTER STRUCTURE}

\subsection{Architecture of MicroPhotonic high-Q RF filter:}

Figures 1(a) shows the proposed architecture of MicroPhotonic RF filter. A low-noise amplifier (LNA) pre-amplifies the input RF signal to compensate for subsequent splitting loss and also to boost the modulation efficiency of the VCSEL array (where the first E stands for "external"). The RF splitter equally splits the input RF signal into N RF signals, which modulate the $\mathrm{N}$ elements of the $1 \times \mathrm{N} 850 \mathrm{~nm}$ VCSEL array integrated on the VCSEL/photoreceiver chip[9, 10]. A VCSEL array can generate high optical power per element while maintaining fundamental mode oscillation. The diffractive optical element (DOE) [11]collimates and routes the Gaussian beams generated by the VCSEL array. Each RF-modulated collimated optical beam generated by a VCSEL element propagates within the optical substrate and undergoes several reflections in a cavity whose width defined by one of the mirrors assigned to that VCSEL element and the DOE. Every time a beam bounces on the DOE, a small fraction of the power of that beam is transmitted through the DOE for detection and amplification by an element of the wideband photoreceiver array that is integrated on the VCSEL/photoreceiver chip, while the remaining large fraction is reflected and routed to for subsequent delayed photodetection. An RF combiner adds (or subtracts) the amplified RF photocurrents to generate the output RF signal.

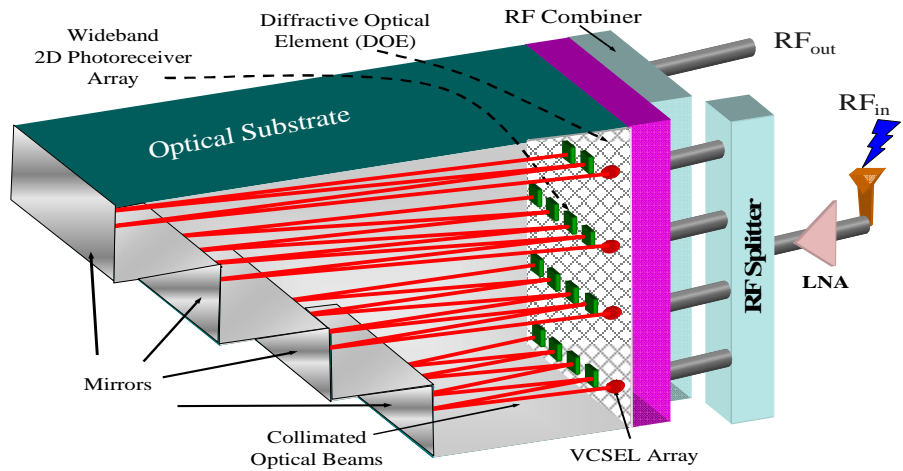

(a)

Figure 1. Architecture of MicroPhotonic RF filter.

\subsection{Transfer function:}

To determine the transfer function of the proposed MicroPhotonic RF filter, we assume that the input RF signal to the external modulator is a linearly modulated signal of RF frequency $\omega$ given by:

$$
V(t)=1+M \cos (\omega t)
$$

Since the optical input to the external modulator is a continuous wave (CW) signal, therefore, the relationship between the input voltage to the modulator and its output optical power is linear. The relationship between the output electric field from modulator and the applied RF input voltage can also be assumed linear when the optical modulation index, $M$, is small. Under this assumption, the modulator output electric field is given by

$$
E(t) \propto \sqrt{V(t)}=\sqrt{1+M \cos (\omega t)} \approx 1+m \cos (\omega t)
$$

where $m \approx M / 2$. Assume each VCSEL element generates an optical power $P_{0}$, the photocurrent on the $\mathrm{n}^{\text {th }}$ photodetector of the $\mathrm{m}^{\text {th }}$ cavity is given by:

$$
I_{n, k}=P_{n} \mathfrak{R}\left(1+\eta_{V C S E L} \cdot \cos \left(\omega t+n T_{k}\right)\right)
$$

where $\mathfrak{R}$ is the photoreceiver responsivity and $\eta_{V C S E L}$ is the modulation response of the VCSEL elements, and $P_{n}$ and $T_{k}$ are given by

$$
\begin{aligned}
& \left.P_{n}=P_{0}(1-R) R^{n-1}\right) \\
& T_{k}=\frac{\sqrt{4 L_{k}^{2}+d^{2}}}{v}
\end{aligned}
$$

Note that $P_{n}$ is the optical power of signal after being sampled $n$ times in a cavity, $R$ is the reflectivity of DOE. $T_{k}$ and $L_{k}$ are the sampling time and length of the $k^{\text {th }}$ cavity, respectively, $d$ is the spacing between the photoreceiver elements, and $v$ is the speed of light inside the cavity. By adding the photocurrents, the output current of the RF combiner becomes: 


$$
I(t)=\sum_{k=0}^{N-1} \sum_{n=0}^{N-1} G_{n, k} I_{n, k}\left(t+n T_{k}\right)
$$

where $G_{n, k}$ is the gain of the photoreceiver element $(n, k)$. Since the output signal is the summation of photocurrents generated by the photoreceiver array, therefore the filter's response is free of phase-induced-intensity noise which is caused by high coherence laser source. The transfer function of the filter is therefore given by:

$$
H(\omega)=\sum_{k=0}^{N-1} \sum_{n=0}^{N-1} \Re \eta_{V E C S E L} P_{0}(1-R) R^{n} G_{n, k} \exp \left(-j n \omega \frac{\sqrt{4 L_{k}^{2}+d^{2}}}{v}\right)
$$

By optimizing the gains of the photoreceiver array an arbitrarily Finite Impulse Response (FIR) can be synthesized thus realizing a reconfigurable transversal $\mathrm{RF}$ filter.

\subsection{Q factor:}

A typical transversal filters spectral response is shown in Figure 2. The $\mathrm{Q}$ factor, or quality factor, is defined as:

$$
Q=\frac{F S R}{\Delta f_{F W H M}}
$$

Where $\Delta \mathrm{f}_{\text {FWHM }}$ is the frequency width at half maximum power of the passband and FSR is the free spectral range of the filter. The FSR depends on the sampling frequency $f_{c}=1 / F S R$. It is important to obtain a high Q-factor in some applications where information channels to be selected or filtered out are very closely spaced.

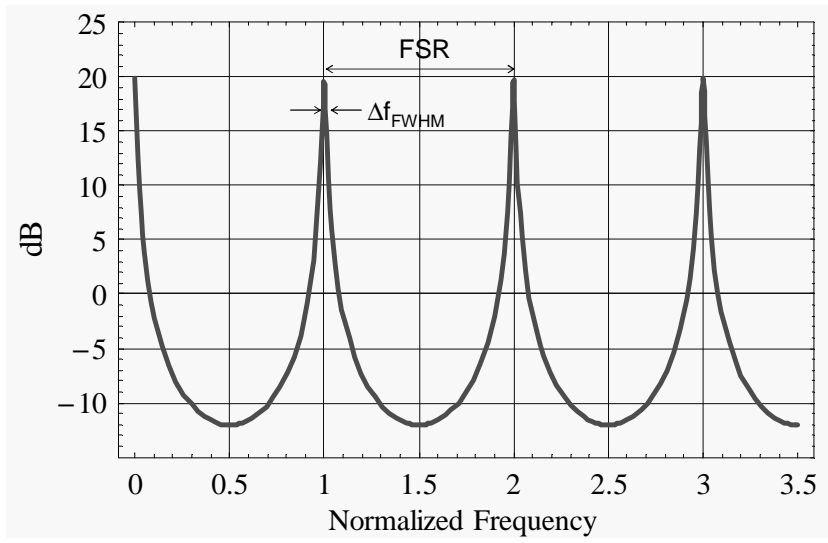

Figure 2: Typical response of a transversal bandpass filter.

\subsection{Gaussian beam divergence control within the cavity.}

In order to design a reconfigurable high-resolution RF filter, it is necessary to generate a large number of taps within the cavity. Since the laser beam is Gaussian, it undergoes significant divergence as it propagates inside a cavity. Beam divergence leads to crosstalk which limits the maximum propagation length and hence the number of taps. The Gaussian beam radius $\omega(x)$ and the wavefront radius of curvature $\mathrm{R}(\mathrm{x})$ are given by:

$$
\begin{aligned}
& \omega^{2}=\omega_{0}^{2} \cdot\left(1+\left(\frac{\lambda \cdot x}{\pi \omega_{0}^{2}}\right)^{2}\right) \\
& R(x)=x \cdot\left(1+\left(\frac{\lambda \cdot x}{\pi \omega_{0}^{2}}\right)^{2}\right)
\end{aligned}
$$

where $\omega_{0}$ is beam waist at $x=0$ and $\lambda$ is the wavelength. 


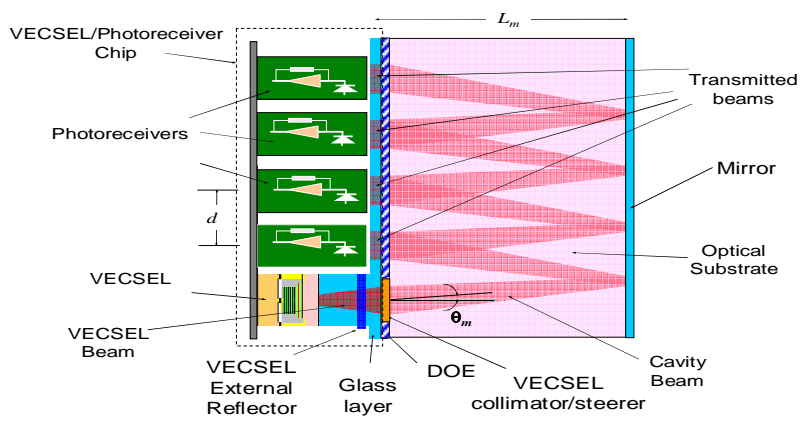

Figure 3. Interface between the VCSEL/photoreceiver chip and the optical substrate.

To keep the crosstalk below an allowable level (practically $<-30 \mathrm{~dB}$ ), a DOE film can be inserted between the VCSEL/photoreceiver chip and the optical substrate to appropriately focus the diverging beam and maintain its diameter within an acceptable range. Figure 3 shows the interface between the VCSEL/photoreceiver integrated chip and the optical substrate, and a typical optical beam propagating within the optical cavity. The DOE can be appropriately designed in such a way that as the optical beam hits the DOE, a large portion of its power is reflected inside the optical cavity while a small fraction of its power is transmitted through the DOE and detected by one of the photoreceivers.

\subsection{Simulation results:}

\section{RESULTS}

Figure 7(a) shows the responses of a high-Q bandpass filter of a 32-cavity MicroPhotonic filter employing a $32 \times 32$ photodetector array for different centre frequencies. It is shown that the centre frequency of the filter can be adapted by optimizing the gain profile of the photoreceiver array. A bandwidth as small as $5 \mathrm{MHz}$ with centre frequency of $2.5 \mathrm{GHz}$ is displayed in Fig. 7(a), which corresponds to a $\mathrm{Q}$ of 500. The sidelobe rejection of the filter is more than $25 \mathrm{~dB}$. In Figure 7(b) the filter centre frequency is coarsely tuned from $0.5 \mathrm{GHz}$ to $4.5 \mathrm{GHz}$. Note that sampling frequency was 10 $\mathrm{GHz}$ which corresponds to a minimum optical substrate thickness of $10 \mathrm{~mm}$.

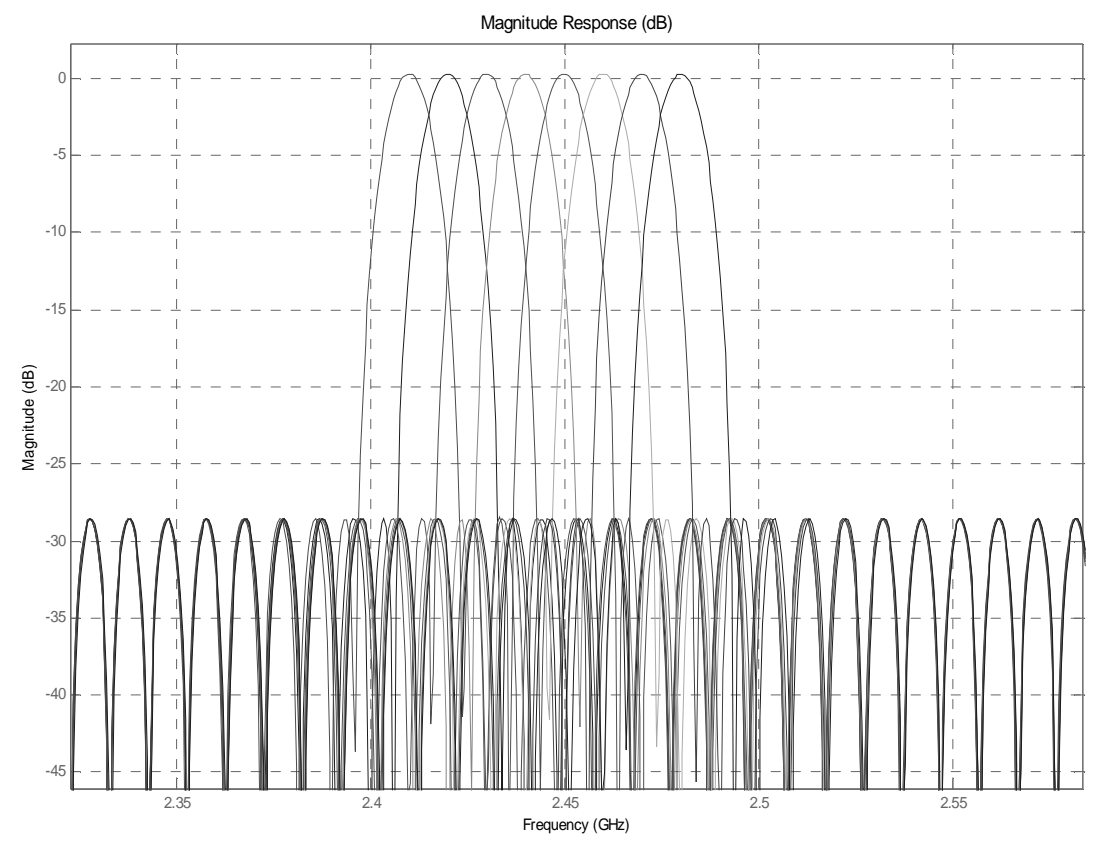

(a) 


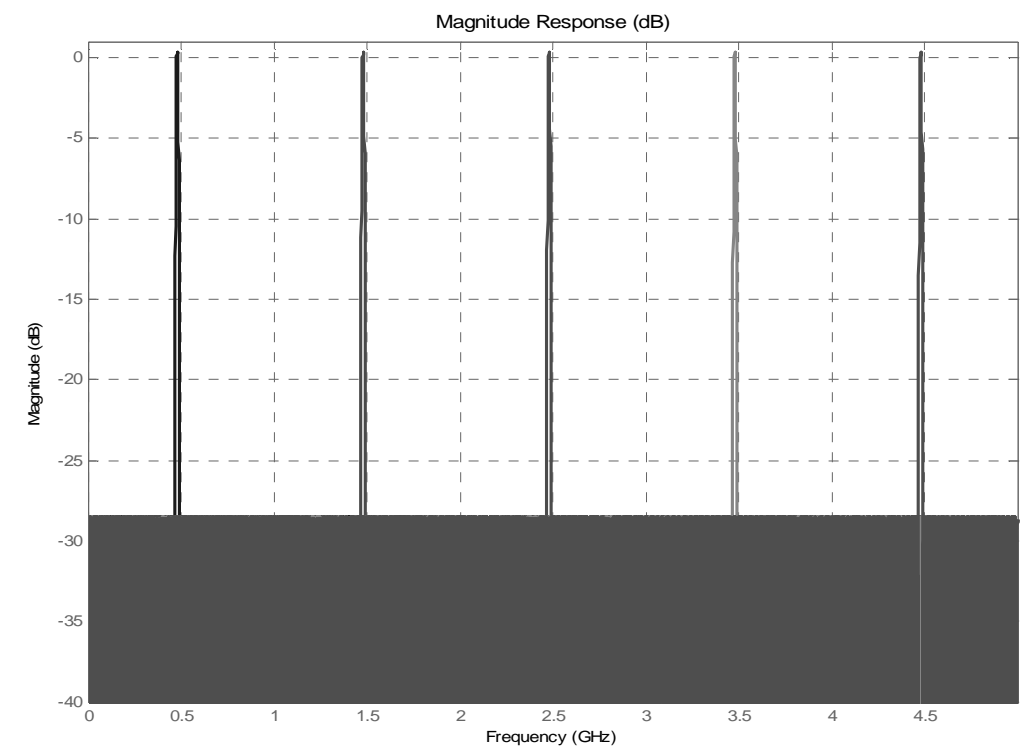

(b)

Figure 7. Adaptive high-Q filter responses: (a) Fine tuning around $2.5 \mathrm{GHz}$, (b) Coarse tuning.

Figure 8(a) shows a dual-passband response of the MicroPhotonic filter, together with the corresponding gain profile, which was optimized to realize two passband at $2.5 \mathrm{GHz}$ and $7.7 \mathrm{GHz}$. In Figure $8(\mathrm{~b})$, the weights of taps was optimized so that one of the passbands is kept at $2.5 \mathrm{GHz}$, while the other passband is tuned to $5.6 \mathrm{GHz}$. This demonstrates the capability of the MicroPhotonic RF filter to synthesize adaptive multi-bandpass responses over a wide frequency band. The "remez" algorithm [12] was used to determine the optimum coefficients of the MicroPhotonic FIR filter.
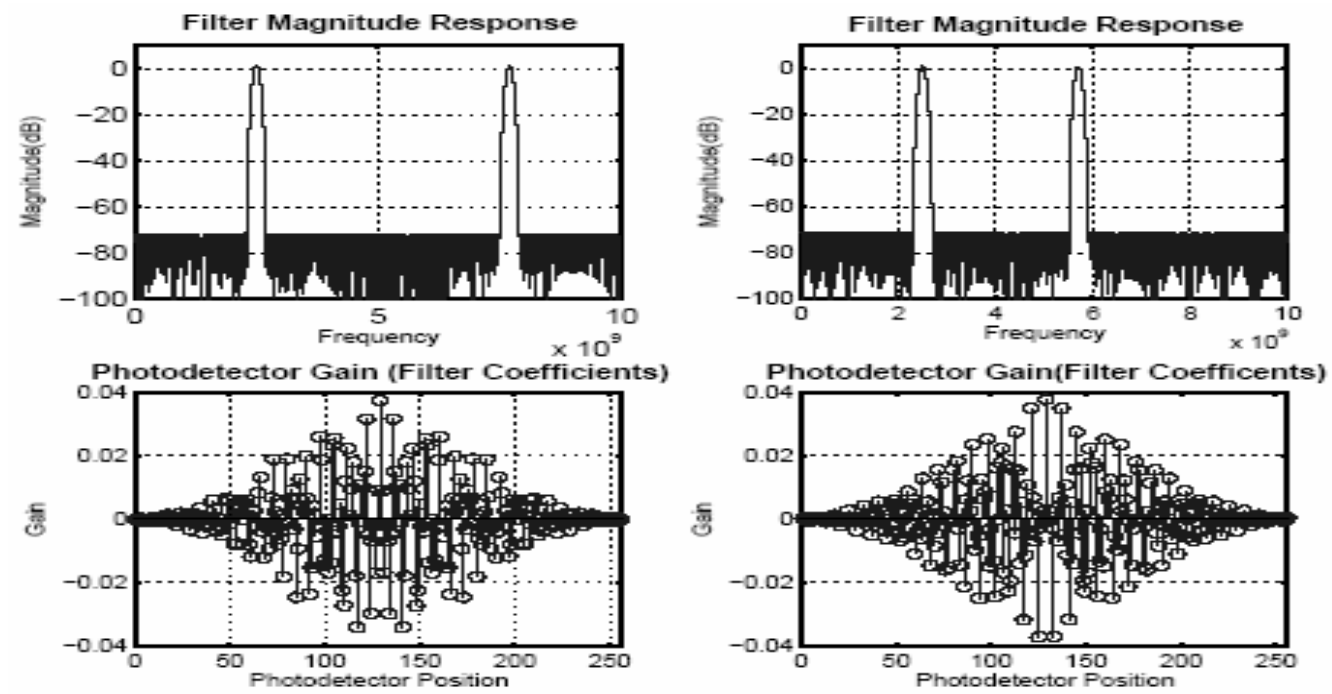

(a)

(b)

Figure 8. MicroPhotonic high-Q filter: (a) Magnitude response. (b) Filter coefficients.

\subsection{Experimental results:}

To prove the principle-of-concept for the MicroPhotonic filter, a 4-tap filter was built by using 4 identical discrete Ortel 2601B photoreceivers of bandwidth $200 \mathrm{MHz}$ and optical fibers as RF true-time delay lines, as shown in Figure 9(a). Since the number of available photoreceivers was limited to 4 , it was easier to generate a high-Q notch response rather than a bandpass response, we synthesized a notch response with a centre frequency around $200 \mathrm{MHz}$. The incremental optical fiber lengths were chosen to be $2 \mathrm{~m}, 4 \mathrm{~m}$, and $6 \mathrm{~m}$. Figure 9 (b) shows the measured and predicted normalized 
responses of the 4-tap MicroPhotonic filter. The weights of the MicroPhotonic interference rejection filter were optimized to realize a notch depth of more than $35 \mathrm{~dB}$. An excellent agreement between the measured and predicted frequency response for the MicroPhotonic interference rejection filter can be seen. Note that the slight discrepancy between the measured and predicted responses at high frequencies is due to the roll-off of the filter beyond $200 \mathrm{MHz}$.

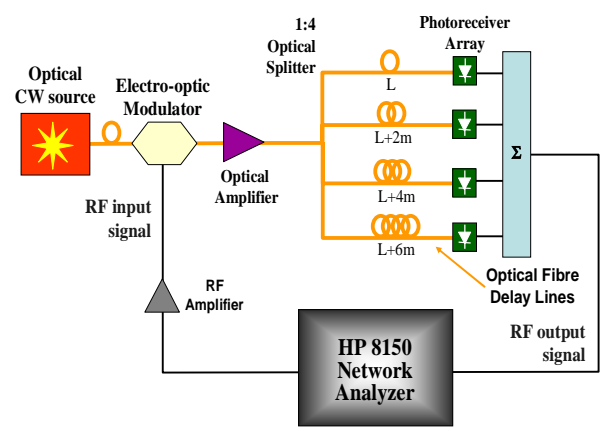

(a)

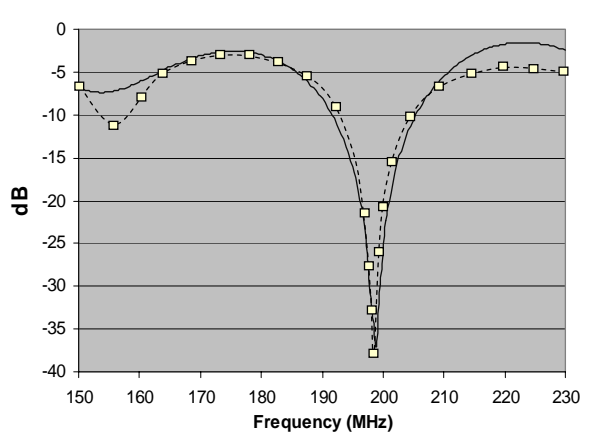

(b)

Fig. 9 (a) 4-tap MicroPhotonic RF filter. (b) Measured (squares) and predicted (solid line) RF responses.

Figures 10(a), (b) and (c) show measured responses of the MicroPhotonic RF filter, where the gains of the photoreceivers were optimised to generate notch responses of centre frequencies $158 \mathrm{MHz}, 193 \mathrm{MHz}$, and $212 \mathrm{MHz}$, respectively. These demonstrate the reconfigurability of the MicroPhotonic filter and its ability to tune the centre frequency through reconfiguration of the gain profile of the photoreceiver array.

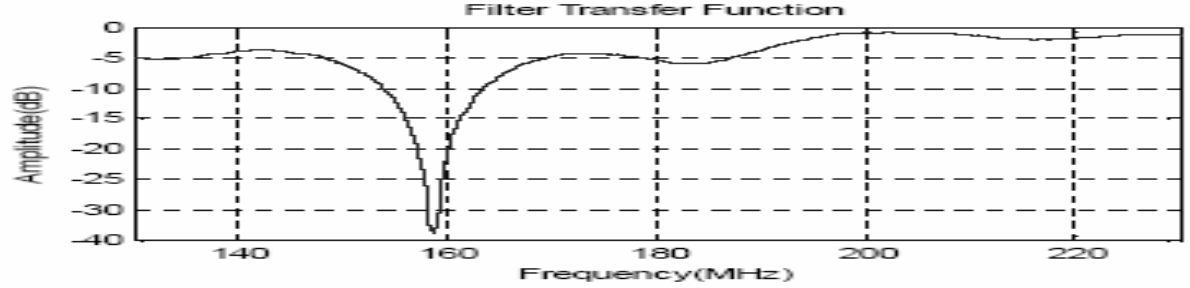

(a)

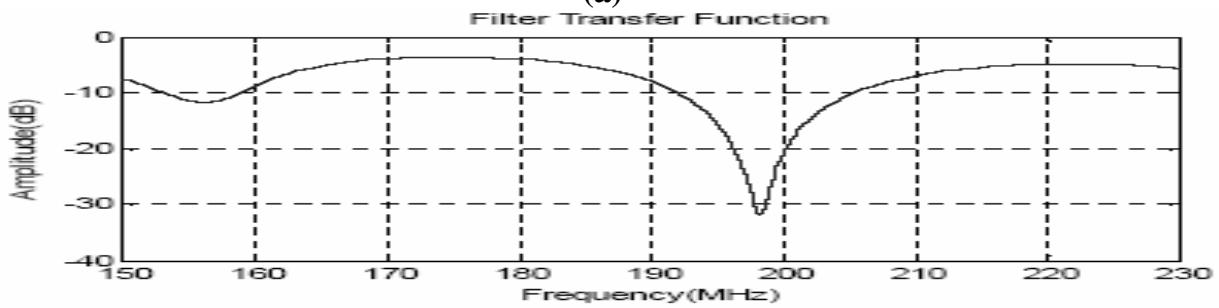

(b)

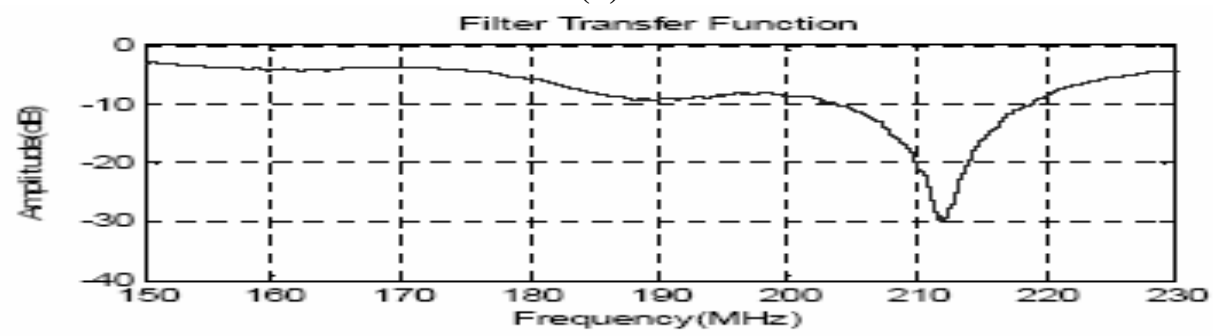

(c)

Fig.10. Measured responses of the MicroPhotonic RF filter for different notch frequencies: (a) $158 \mathrm{MHz}$, (b) $197 \mathrm{MHz}$, (c) $212 \mathrm{MHz}$. 


\section{CONCLUSIONS}

A novel MicroPhotonic architecture integrating a photoreceiver array, a Vertical Cavity Surface Emitting Laser array, and a multi-cavity optical substrate to realize a low-cost tunable high-Q bandpass RF filter, has been presented. Simulation results showed that for a 32-cavity MicroPhotonic structure, a tuneable bandpss RF filter with a Q factor as high as 500 is feasible. It was also shown that a multi-band RF bandpass filter can be realized with the center frequency be tuned adaptively. A 4-tap proof-of-concept MicroPhotonic filter was experimentally demonstrated. Excellent agreement between simulation and experiment was observed, and by reconfiguring the gain profile of the photoreceiver array, tuning of the filter center frequency was experimentally realized.

\section{REFERENCES}

1. Matthews, P.J., Biernacki, P.D., "Photonic signal processing for microwave applications", 1999 IEEE MTT-S international microwave symposium digest, vol. 3, pp. 877-880, 1999.

2. Frankel, M.E. and R.D. Esman, "Fiber-optic tunable microwave transversal filter", IEEE Photonics Technology Letters, 7(2): p. 191-193, 1995.

4. Demestichas, P., et al., "Evolution in wireless systems management concepts: from composite radio environments to reconfigurability", IEEE Communications Magazine, 42(5): p. 90-98, 2004.

5. Leaves, P., et al., "Dynamic spectrum allocation in composite reconfigurable wireless networks", IEEE Communications Magazine, 42(5): p. 72-81, 2004.

6. Hunter, D.B. and R.A. Minasian, "Photonic signal processing of microwave signals using an active fibre bragggrating-pair structure", IEEE Transactions on Microwave Theory and Techniques, 45(8): p. 1463-1466, 1997.

7. You, N. and R. Minasian, "High-Q optical microwave Filter", Electronics Letters, IEE, 35(24): p. 2125-2126, 1997.

8. Capmany, J., D. Pastor, and B. Ortega, "New and flexible fiber-optic delay-line filters using chirped Bragg gratings and laser arrays", IEEE Transactions on Microwave Theory and Techniques, 47(7): p. 1321-1326, 1999.

9. Chen, Y. and R.T. Chen, "A Fully Packaged True Time Delay Module for a K-band Phased Array Antenna System Demonstration", IEEE Photon. Technol. Lett., vol. 14, pp. 1175-1177, 2002.

10. Morgan, R.A. "VCSEL research, development and applications at Honeywell", in Vertical-Cavity Lasers, Technologies for a Global Information Infrastructure, WDM Components Technology, Advanced Semiconductor Lasers ..., Gallium Nitride Materials, Processing, ..., 1997 Digest of the IEEE/LEOS Summer Topical Meetings. 1997.

11. Kosaka, H., "Smart integration and packaging of 2D VCSEL's for high-speed parallel links", IEEE Journal of Selected Topics in Quantum Electronics, 5(2): p. 184-192, 1999.

12. Fu, Y., N. Kok, and A. Bryan, "Design of hybrid micro-diffractive-refractive optical element with wide field of view for free space optical interconnections", OPTICS EXPRESS, 10(13): p. 540-549, 2002.

13. J S. Lim and A V. Oppenheim, "Advanced Topics in Signal Processing", 1988: Englewood Cliffs, NJ: PrenticeHall. 